\title{
Grandparents and Social Work Services in Focus in Tehran, Iran: Gerontological Perspectives
}

\author{
Mohammad Taghi Sheykhi* \\ Department of Social Science, Iran \\ *Corresponding author: Mohammad Taghi Sheykhi, Professor of Sociology, Department of Social Science, Tehran, Iran \\ Submission: 監 September 12, 2017; Published: 望㿼 December 21, 2017
}

\begin{abstract}
The aim of this article is to represent how the elderly as an increasing demographic group are in need of related social work services. For that, new social policies must be designed and put into practice. As the number of the elderly people is ever increasing, and generally one party/spouse dies at his/her old age, social work services become vital for the remaining party. The growing elderly expect to benefited from different social and welfare benefits at older age when they are weak, poor, alone and unhealthy. This is not only the problem of Iran, but almost majority of developing countries are in this situation. Such people need increasing number of social and medical services which must be usually provided by social workers. However, the rising costs of social and health services unable the elderly and grandparents to utilize them; hence social workers need to intervene to provide what is needed. In addition to the necessary needs, the elderly grandparents need recreational services as well.
\end{abstract}

Keywords: Social work; Social policies; Grandparents; Old age; Medical services

\section{Introduction}

Social work is concerned with individual and personal problems; similarly with boarder social issues such as poverty, unemployment, domestic violence etc. The concept is used by social science researchers in various perspectives. However, the most important domain of social work affecting quality of life is the overall satisfaction of individuals with life.

Iran with a population of over 79.5 million in mid-2016 [1] is facing increasing older population because of more than 62 percent of population at age groups 15-64. Such a state is creating vulnerabilities as far as grandparent's vs social work services are concerned. The gap between the needs of grandparents and how they are met is provided by the professional social workers. Social tensions are also minimized through social work services.

Rapid increase in the proportion of the elderly in modern societies [2] is considerably noticeable in Iran, where the percentage of people $65+$ has almost doubled in 50 years from $4 \%$ in 1956 to $8.3 \%$ in 2011 [3]. One reason for this surge could be the accessibility to social and medical services by the elderly that include grandparents too. It is well established that the elderly consume large share of the health-care services in modern societies [4]. While due to demographic change the number of the elderly people and grandparents is increasing with increasing needs and expectations, adequate number of social workers is not available to comply with such needs in social, family and medical contexts. Under such circumstances the elder grandparents are abused. They even sometimes lose their self-esteem.
Because of the new lifestyle and new form of family style, social work services need to be provided to fill the vacuum. However, duties carried out by social workers vary depending on the settings in which they perform duties to assist grandparents. These negative attitudes may in turn generate negative, stereotyped, dependent behaviour among the elderly [5]. To meet the increasing elderly citizens' needs, more professional social workers must be trained.

As a whole, the amount and quality of help requested by the grandparents of different backgrounds is not the same. Social work institutions, have thus far failed to plan for the changing shape of the population, so agencies and services are being adapted in the midst of overwhelming demands [6].

Though there are very few sources and scientific documents on grandparents in Iran, the author is trying to create views and literature on the topic by collecting data and using relevant materials through referring to foreign resources. "Third Age" appearing as a new phenomenon, it is highly depended on the functions of social work to help grandparents and families to solve their emerging problems which affect their performance.

While in the past, grandparents were very influential over the youth, social structure of the family was such that, almost every decision-making of the younger generations was directed by the authority of grandparents [7] yet, in the course of time, and due to the increasing education and socialization of the children and youth, the grandparents' authority over the grandchildren has declined. Therefore, social work intervenes to create a better safety 
and quality of life for both families and grandparents. The present work tries to reflect a perspective of grandparents with special reference to their own lives, i.e. their safety and quality of life (Table 1).

Table 1: Comparative Profile of the Number and Proportion of "Potential" Grandparents 65 years and over in Iran and in Tehran City Between 1956 and 2011.

\begin{tabular}{|c|c|c|c|c|c|c|c|}
\hline \multicolumn{3}{|c|}{ Iran } & \multicolumn{3}{c|}{ Tehran City } \\
\hline Year & Population & (Potential) Grandparents & \% Total & Year & Population & (Potential) Grandparents & \% Total \\
\hline 1956 & 18954704 & 758670 & 4 & 1956 & 1560434 & 52013 & 3.3 \\
\hline 1966 & 25788722 & 993045 & 3.8 & 1966 & 2719730 & 81295 & 3 \\
\hline 1976 & 33708744 & 1186470 & 3.5 & 1976 & 4536264 & 14829 & 3.3 \\
\hline 1986 & 49445010 & 1493382 & 3 & 1986 & 6010075 & 203062 & 3.4 \\
\hline 1996 & 60055488 & 2587437 & 4.3 & 1996 & 6758845 & 320430 & 4.6 \\
\hline 2006 & 70472846 & 5130000 & 7.3 & 2006 & 7803883 & 464638 & 6 \\
\hline 2011 & 75149669 & 4343041 & 5.8 & 2011 & 8154051 & 604283 & 7.5 \\
\hline
\end{tabular}

In general, due to increasing facilities, the elderly have found it to live longer life, and remain longer in married life, letting them eventually appear as grandparents. In these settings the social workers contribute to the care, treatment and rehabilitation of the aged as well as care of the disabled persons. Years of living together contributes to mutual understanding and companionship to likely increase between old couples, or what is known in this paper as grandparents. By about age fifty, most couples have completed the task of raising children. From this age on, the couples gradually enter the period of grandparenthood. The remaining years of marriage - "the empty nest" brings a return to living with only one's spouse, and a decline in their socialization.

In health centres, social workers are involved in the provision of counselling to individuals or families and in providing services to the seniors. They also help citizens to identify their needs and propose ways necessary to meet those needs. Those born in 1950s-now in their fifties, will spend as many years looking after their aging parents, as they did caring for their own children [8].

Retirement also brings a change to family life. If the spouse has been a homemaker, the husband's retirement mean that spouses will spend much more time together. Although the husband's presence is often a source of pleasure to both, more in the Western world, it sometimes undermines wives' established routines to the point of intrusion [9]. In post retirement life, social workers further regulate the relations of grandparents who have to spend more time together. They principally need such counsellors to reunite them.

The most influential and tragic transition in the married life appears after one spouse dies. Wives who usually outlive their husbands, needs to be tolerant enough, and that can happen through the counselling of social workers. Under such conditions, they expect to spend a significant period of their lives as widows. Loneliness accompanying the death of a spouse is always difficult. This experience may be even harder for grandfathers or widowers, who usually have fewer friends than grandmothers or widows [10]. In the present research, variables such as age, state of life, state of occupation, income, welfare, insurance, safety, education etc. have been investigated among the grandparents.

\section{Research Design}

The problem of research not touched before in Iran, and being socially very important, made the researcher intervene and appraise the relevant relations in Tehran City. The research design in this work involves identification and selection of research problem, choice of theoretical framework (conceptual model) for research problem. In that, sampling procedure, tools and techniques of gathering data, processing of data, analysis of data, presentation, discussion and interpretation of data, and generalization of research findings were all thought of.

\section{Method of Research}

The author first reviewed the necessary and background literature and theories on aging grandparents. In the theoretical section, relevant theories were searched, and the perspectives necessary were extracted, and used in the text. While the aging grandparents are not easily approachable, yet the researcher was patient enough to find them in families where they used to live. The researcher could refer to the intended samples in all the 22 different municipal parts of the city.

The empirical section of the research included 452 samples of age 65+. They were randomly selected in various neighbourhoods of Tehran City and for whom the questionnaires were filled in. The selected grandparents were interviewed too, in the course of referrals. Eventually, the questionnaires were edited, electronically extracted and tabulated in the form of designed tables. The main hypothesis of the research is: Provision of social work services leads to improved quality of life of grandparents.

\section{Findings}

Based on preliminary identification, those elderly who were really grandparents were identified, and for whom the face-toface questionnaires were administered. In the process of referral, the researcher could find 272 living grandfathers and 402 living grandmothers. That is, a sex ratio of 67.7 (Sex Ratio $=\times \mathrm{K}$ ), which 
is natural and normal according to the Iranian demographic standards.

The author recognized how effective social workers are in the lives of older grandparents, and in various perspectives. They can influence and improve their quality of life. While such a study has not so far been done on older grandparents in Iran, the results could be generalizable to other parts of Iran, since social work is poorer in the rest of the country.

After data tabulation, the researcher reached more than 50 tables which was impossible for him to reflect all. Therefore, the data and tables have been analyzed and highlighted in the form findings.

In referral to families to screen/find out about different characteristics of grandparents, the researcher could find 272 a live grandfathers against 402 alive grandmothers, i.e. a sex ratio of 68 males for every 100 females or grandmothers. Within those alive, $75 \%$ used to live together, while $25 \%$ of those elderly couples used to live with their offspring. In another query, the author came to know that $60 \%$ of sample grandfathers lived alone, while $40 \%$ used to live with their offspring. In this query, $73 \%$ of grandmothers used to live alone, while $27 \%$ used to live with their offspring. Similarly, occupational status of grandfathers was as such: $27 \%$ were busy in jobs, $41 \%$ pensioners, $1 \%$ had no pension at all, $7 \%$ were depended on offspring and finally $24 \%$ were depended on their own wealth. In this regard, only $2.75 \%$ of grandmothers were engaged in jobs, $35.78 \%$ were pensioners, $15.6 \%$ had no pensions, $18.35 \%$ were depended on their offspring and $27.52 \%$ were depended on their own wealth.

Income being an important factor in old age, it was found that: $14 \%$ of grandfathers asserted to earn about 107 US \$ a month, $16.5 \%$ declared to have monthly income of about US $\$ 100$ and 160 . Those having monthly income of US $\$ 160$ and 180 were $21 \%$, grandfathers with monthly income of US \$180 and 267 demonstrated $20.5 \%$, and finally $28 \%$ declared to have an income above US $\$ 268$ a month. So far as the grandmothers' income status is concerned, they declared their monthly income as such: $35.36 \%$ had income of less than US $\$ 107$ per month, 19\% of grandmothers had income of between US $\$ 107$ and $160,15.22 \%$ had monthly income of US \$ 160 and $180,14.07 \%$ had monthly income of US $\$ 180$ and 267 , and finally $16.35 \%$ of grandmothers declared to have a monthly income of US $\$ 268$ and above.

While insurance plays a determining role in the safety and security of the ageing grandparents, $82.72 \%$ of our sample grandfathers declared to have insurance and the rest of $17.28 \%$ declared not to be under any health insurance coverage. In a query regarding the health insurance of grandmothers, $84 \%$ had insurance, and $16 \%$ did not have it.

In another table prepared to find out about the health conditions of grandfathers the data collected were as follows: $36.03 \%$ were healthy, $11.75 \%$ had arthritis, $20.22 \%$ had some heart disease, $7 \%$ had diabetes, and $25 \%$ had other diseases. In case of grandmothers, $26.62 \%$ were healthy, $28.61 \%$ had arthritis, $12.94 \%$ had some heart diseases, $12.19 \%$ had diabetes, and 19.65 had other diseases.

Older generations are not quite educated in Iran. Therefore, a perspective of the educational status of grandfathers could be reflected as follows: $26.84 \%$ uneducated, $30.88 \%$ had only primary education, $12.5 \%$ education of below ninth grade, $24.27 \%$ had finished secondary school or below, $1.84 \%$ had finished a diploma or college degree, $1.84 \%$ had a B.A./ B.Sc., and also $1.83 \%$ had a master's degree or above. Educational reflections on grandmothers are a bit different from those of grandfathers. Table concerning grandmothers states that $46.02 \%$ of them were illiterate, $30.6 \%$ had primary education, $6.47 \%$ had education of below ninth grade, $13.33 \%$ had finished secondary school or below, $2.24 \%$ had finished a diploma or a college degree, $1 \%$ had a B.A, B.Sc and only $0.25 \%$ of the sample grandmothers had master's or above.

Classification of grandfathers according to their general feelings could be explained as such: $19.12 \%$ of our sample grandfathers declared to have loneliness feeling, $44.12 \%$ had impatience or moodlessness feelings, $7.72 \%$ had feelings of nonassistance or helplessness, $12.5 \%$ feared death, and finally $16.54 \%$ of grandfathers declared no remarks. In case of grandmothers, feeling of loneliness among them was $30.35 \%$, impatience was $30.6 \%$, feeling of non-assistance $14.43 \%$, fear of death was $12.19 \%$, and $12.44 \%$ declared no remarks.

Another table indicating grandfathers' status could be reflected as follows: $10.66 \%$ were in poverty, $29.41 \%$ were in social isolation, $30.15 \%$ suffered insecurity, and $29.78 \%$ gave no comments. Similarly, grandmother respondents were found, 9.2\% in poverty, $25.87 \%$ in isolation, $35.82 \%$ in insecurity, and $29.1 \%$ with no comments.

In a query regarding the satisfaction of grandfathers, various reflections were found accordingly, i.e. 59.19\% were satisfied with their lives, $18.75 \%$ were not satisfied with their lives, $8.82 \%$ expressed happiness, and finally $13.24 \%$ expressed grief. In case of grandmothers, $49.5 \%$ were satisfied with life, $16.17 \%$ were dissatisfied with their lives, $9.95 \%$ were happy, and $24.38 \%$ were grieved.

Similarly, attitudes of grandfathers towards different conditions were measured as such: $54.04 \%$ were satisfied with their marriage, $6.25 \%$ were discontented with their marriage, $5.15 \%$ were satisfied with their income, $10.66 \%$ were discontented with their income, $11.03 \%$ of the grandfathers were satisfied with the atmosphere/ their surroundings, and finally $12.87 \%$ of the grandfathers were discontented with their atmosphere around. In terms of grandmothers, the data showed that $35.82 \%$ were satisfied with their marriage, $10.7 \%$ were discontented with their marriage, $3.48 \%$ were satisfied with their income, and $15.17 \%$ were discontented with that. Similarly, $15.17 \%$ of grandmothers studied were satisfied with the atmosphere around them, and 19.65\% were dissatisfied with that.

Other indicators pertaining to the way of life of the grandparents and approaches towards them in the family are reflected in a compact table as follows (Table 2): 
Table 2: Multi-indicator Perspective of Grandparents in Tehran.

\begin{tabular}{|c|c|c|c|c|c|}
\hline \multicolumn{6}{|c|}{ The Way Grandparents are Approached in the Family } \\
\hline \multirow{2}{*}{ Grandfathers } & $100 \%$ & With justice & With affection & \multicolumn{2}{|c|}{ With discrimination } \\
\hline & & $34.19 \%$ & $52.94 \%$ & \multicolumn{2}{|c|}{$12.87 \%$} \\
\hline Grandmothers & $100 \%$ & $16.42 \%$ & $68.66 \%$ & \multicolumn{2}{|c|}{$14.93 \%$} \\
\hline \multicolumn{6}{|c|}{ The way the ideas/experiences of grandparents are used in the family } \\
\hline \multirow{2}{*}{ Grandfathers } & $100 \%$ & \multicolumn{2}{|c|}{ Use of ideas } & \multicolumn{2}{|c|}{ Non-use of ideas } \\
\hline & & \multicolumn{2}{|c|}{$69.85 \%$} & \multicolumn{2}{|c|}{$30.15 \%$} \\
\hline Grandmothers & $100 \%$ & \multicolumn{2}{|c|}{$61.69 \%$} & \multicolumn{2}{|c|}{$38.31 \%$} \\
\hline \multicolumn{6}{|c|}{ Safety and psycho-mental dependency of grandparents } \\
\hline \multirow{2}{*}{ Grandfathers } & $100 \%$ & Visiting relatives & Visiting friends & Visiting offspring & None \\
\hline & & $26.47 \%$ & $10.66 \%$ & $49.26 \%$ & $13.60 \%$ \\
\hline Grandmothers & $100 \%$ & $27.61 \%$ & $6.47 \%$ & $59.45 \%$ & $6.47 \%$ \\
\hline \multicolumn{6}{|c|}{ Safety dependency of grandparents } \\
\hline \multirow{2}{*}{ Grandfathers } & $100 \%$ & Adequate income & Care by offspring & \multicolumn{2}{|c|}{ Care by nurse } \\
\hline & & $42.65 \%$ & $55.88 \%$ & \multicolumn{2}{|c|}{$1.47 \%$} \\
\hline Grandmothers & $100 \%$ & $27.61 \%$ & $70.15 \%$ & \multicolumn{2}{|c|}{$2.24 \%$} \\
\hline \multicolumn{6}{|c|}{ Priority of grandparents' lives } \\
\hline \multirow{2}{*}{ Grandfathers } & $100 \%$ & Living alone & Living in birth place & Living with spouse & Living with affspring \\
\hline & & $6.99 \%$ & $22.06 \%$ & $54.04 \%$ & $16.91 \%$ \\
\hline Grandmothers & $100 \%$ & $10.45 \%$ & $14.18 \%$ & $41.29 \%$ & $34.08 \%$ \\
\hline \multicolumn{6}{|c|}{ Grandfather dead and state of life of grandmother } \\
\hline \multirow{2}{*}{ Grandfathers } & $100 \%$ & \multicolumn{2}{|c|}{ Living alone } & \multicolumn{2}{|c|}{ Living with offspring } \\
\hline & & \multicolumn{2}{|c|}{$72.78 \%$} & \multicolumn{2}{|c|}{$27.22 \%$} \\
\hline Grandmothers & $100 \%$ & \multicolumn{2}{|c|}{$60 \%$} & \multicolumn{2}{|c|}{$40 \%$} \\
\hline
\end{tabular}

\section{Discussion}

Population ageing in Iran as in most other countries is an unprecedented phenomenon. As predicted, by UN, population aged 65 and over will outnumber those aged below 15 by the year 2050 in most developed societies and the European countries [11]. As far as Iran is concerned, due to declining birth rates and decline in replacement level indicator, the elderly people will outnumber those under the age 15 by the year 2050 . Such a scenario will result in increasing issues of health care, housing and other social services for the elderly including grandparents in the years to come.

In brief, the long-term decline in fertility and contraction in the family size, are leading, and will further lead to a decrease in the population (0-14), which will in turn push up the population into the working age; followed by increase in elderly population and more grandparents. However, the emerging population change, and the inverted demographic pyramid will lead to increasing problems which need adequate social work services for the elderly grandparents.
In Iran, services and supports for the elderly are mainly provided by four government organizations, i.e. Imam Khomeini Relief Committee, Islamic Republic Martyr's Foundation, State Retirement Organization, and the State Welfare Organization.

\section{Services provided by these organizations in 2011 are as follows}

About 150.000 elder rural persons are paid pensions through Shahid Rajaee Project. In Iran, a new welfare project started in 2005 for the elderly called Manzelat (Respect) Cards, distributed among the elderly by the State Retirement Organization in the form of Trip Allowance Credit Cards- letting the elderly commute free of charge.

Though families try to help and support grandparents in Iran; to improve their quality of life, yet, such helps/services are inadequate. Therefore, government and public services in favour of the aging people are urgently needed to be implemented. Similarly, loneliness, nursing, sickness, depression, and health care are major emerging problems of the aging grandparents that require 
immediate solutions. The scenario could highly be helped by the government policies.

The large number of older people in Iran as in many other countries is a new phenomenon, and according to [12], it calls for invention rather than imitation. Therefore, progressive ways and solutions must be investigated in order to improve old age today.

The researcher has attempted to prove that social work and the new concept of grandparents are much interlinked with special reference to the 21st century. This is where the dilemma begins, and the need for social work services well-felt. However, there is clearly room for an alternative interpretation of roles to be played by the social work towards grandparents.

Grandparents can be of great help to their children. They can influence a family's adjustment, and often provide support to the entire family, especially those who are under the coverage of medical insurance $(82.27 \%)$ and not a burden on their children. They often serve many purposes of their grandchildren that their parents cannot fulfil. To be effective supporters, grandparents must first have their own needs and concerns responded and addressed. They have the potentiality of being each other's best resources. Grandparents everywhere could be the best caregivers for millions of children, especially those whose mothers work outside home. They can also transfer older culture to the "third generation". Therefore, such valuable pieces of experiences and advice must be well protected.

To focus attention on the phenomenon, and provide grandparents with the recognition they deserve, the present research has been started. Grandparents bridge between the last generation(s), and the new generation(s). They transfer old culture and values to the younger generation(s) through their care giving to contribute to their social mobility. The interaction is mutual; while grandparents benefit their grandchildren, they are benefited by them too. While they transfer the old culture to the younger generation, they are updated by them too, and interaction prevents them from being socially excluded. To upgrade quality of life in every society, education, culture and clean environment must be promoted within the youngest and the oldest (grandparents) regardless of class, caste and creed. However, sociologists argue how environmental problems are linked to particular cultural values, economic arrangements and welfare of ageing grandparents [13].

Safety of grandparents depends on many factors such as providing social work and health services to them, and also improving their social, physical and mental well-being. Despite being retired, grandparents may be activated to keep on secondary functions such as keeping their grandchildren in parent- absent homes, and thus maintaining their independence for longer time. However, a century back or so, about half of children died before the age of twenty, and few could live to forty [14]. Under such conditions the chance of having grandparents was very low.

Currently, the number of grandparents is increasing due to increase in longevity and life expectancy as compared with any time before. Under such circumstances strong social security need to be adapted and maintained. In short, we can state: "What goes up must come down" [15]. What they immediately need now is better income, more social security, and better coverage of health insurance. So, increase in old age means more grandparents. The trend is likely to increase up to the year 2020 due to high population growth rate of 1960s. However, as the number and proportion of the elderly people increase, the healthcare system will experience an unprecedented influx of grandparents with physical and mental health problems, i.e. various age-related issues will make their care more complex and necessary.

Different Socio-economic backgrounds provide the elderly grandparents with different state of safety; some groups and classes confront with challenges associated with mental health problems, addiction, Alzheimer's etc. For example, $44.12 \%$ of the sample grandfathers in this study showed impatience. Similarly, prevalence of depression among them is quite on increase in modern time. But, fortunately, as a result of advances in medical sciences, many old grandparents can be treated upon clinicians' diagnosis. That is largely possible in the industrial countries, and less practical in developing societies including Iran.

However, despite all the advances taken place at different rates, and in different societies, many physicians are unlikely to recognize and diagnose mental health problems in older adults in the early stages of Alzheimer's disease. Similarly, if and when diagnosed, it so happens that many patients does not receive the necessary treatment. In case of mental problems physicians and clinicians do not take it as serious and pay less attention, while in case of physical problems, fractures etc, they pay more attention to treat them. Therefore, in contemporary time and in many societies, mental illness and aging problems which simultaneously happen to grandparents are simply ignored.

Factors such as the age of grandparents, and whether they both are alive- living together; all contribute to the quality of life of grandparents in Iran. Similarly, occupational position of grandparents, their pensions, their "age and work", their income and as a whole their economic position affect their quality of life, their health conditions etc. In case of Iran, not all the grandparents hold medical insurance; those who have it, can practically have more immune life. Likewise, educational status of grandparents also narrows the gap between grandparents and grandchildren. Education has much led to increasing longevity, or so to say, it has left behind more grandparents, and in the future the number and proportion will be much higher [16]. But, unfortunately not all the grandparents are educated. While many grandparents are in need of help and emotional supports of their children, or grandchildren, that often does not happen at the current complicated conditions.

\section{Theoretical Perspectives and Literature}

In this section, the researcher refers to a large amount of scientific literature associated with theoretical and research publications, scientific journals, reference books, government reports, policy statements, and other materials about the theory 
practice, and results of scientific inquiries. They involved various public heath disciplines, health services research, environmental health, health education, health sciences, sociology, social work, demography, psychology etc.

Gerontologists use a variety of theories to direct their research and explain their findings. They also use a number of methods that help them study the process of ageing as grandparents. The proper use of these methods ensures that the research comes up with reliable and valid findings. Improper use of them can lead to biased and confusing results. However, researchers in a single study may ask a broad range of questions that give a detailed snapshot of a group of people at one point of time [17].

Though nursing homes and other institutions will never take the place of a person's own home or apartment, yet those with pleasing social services will make it acceptable to elder grandparents.

According to many economic demographers, an ageing population and increase in the number of grandparents lead to negative consequences in terms of growth of output per capita. A decreasing ratio of the working-age population to the total population contributes to the increases of the ratio of dependents (grandparents) to working people and the active members of families. This phenomenon is increasingly appearing in the contemporary world with special reference to the countries with longer life expectancy.

The theory of optimal life-cycle human capital investment that has been developed by Ben-Porath [18], Mince [19] and Becker [20], argues that rapid technological change in the form of education contributes to be almost exclusively concentrated at younger ages, poses challenges for rapidly ageing population or grandparents. Hence, the relationship between age and human capital investment and consequently productivity growth cannot be seen in isolation from organizational and institutional factors. Under these circumstances the number of the grandparents is ever increasing. But, due to poor planning and controversial conditions, large number of ageing grandparents is not in healthy and quality conditions in Iran.

No any economic theory provides a clear view as to how ageing affects productivity. Thus, health limitations tend to reduce employment opportunities of ageing grandparents. In response to lower earnings, potential older grandparents with health limitations are likely to reduce hours of work, and retire at earlier ages. Poor health also changes the grandparents' own assessment of the value of themselves.

Japan provides a good example of grandparents' health and retirement within them. It has the most rapidly ageing population (grandparents) in the world, and currently has the highest proportion of people age 65 and older. In 1980, only 9.1\% of the population of that country was aged 65 and older. By 2004, this percentage had increased to $19.5 \%$, and current projections indicate that in 2025 , about $31 \%$ of the Japanese population will be aged 65 and older. That is, about one-third of population will be grandparents [21]. Hence, many other countries will have larger number and proportion of grandparents in the years to come.

When nations develop, the young often move to cities [22]. The young feel less tied to the land, and feel less obligation to care of the elders. This leaves the aged in rural support [23]. Older workers sometimes return to their home countries after years of working in another country. They have no work, no skills, and no pensions, and will grow old in poverty [24]. Countries with social programs and pension plans in place will need to adapt these programs to serve more older people [25,26]. Therefore, social workers need new theories of ageing to explain the changes taking place in developing countries, and new plans for social change that fit the needs of an ageing world. Solutions that fit Western development countries do not necessarily fit the developing nations [27]. While the developed countries created pensions and other welfare programs for their older citizens [28], this approach requires an economy and political system that supports these programs.

However, many developing societies have neither the social services nor the economic resources to help the elderly poor. But, there are some exceptions such as Hong Kong that can do so. Many of the developing nations cannot afford the housing, health, or welfare services for the elderly that Western countries have set up. The AARP reports that 155 countries had social security programs in 1993that covered about a third of the people aged 60 and over in the world. But, these programs remain unstable and in some cases they have failed [29]. Many older people in these countries face economic and social hardships.

Another perspective emphasizes the consequences of demographic change for long time economic growth. Anderson [30], for example, estimates the effect of population ageing, and appearance of larger number of grandparents on average growth rate which is a down-ward trend. Bloom \& Williamson [31] add that the ratio of the non-working- age population (grandparents), to the working age population between 1965 and 1995; suggesting that a baby-boom generation would create a wavelike pattern of real GDP per capita over time. Bloom and Williamson believe that as baby-boomers increase the head count immediately after birth, they reduce per capita income, and the final incidence is on grandparents who are often pensioners.

Population change denotes that the future economic output in most industrialized countries must be accomplished by a smaller and older labour force. A key question is how this development might affect labour productivity as measured by output per worker [32]. In the view of many economists, an ageing population or increasing grandparents has negative consequences for growth in output per capita. Therefore, countries with such population structures must search alternatives to respond to the shortage of their human labour force (15-64). However, though the number of grandparents is increasing, their economic conditions are at risk in countries like Iran. They are at present highly dependent on their children, and the scenario will be worse in the years to come.

Until recently grandparenthood has been a neglected area of study (Cunningham-Burley, 1986). Academic interest has been 
much more widespread in the USA [33]. Roles that have been identified are surrogate parent [34]. One matter of concern has been the lack of legal rights and obligations that parents have in relation to their grandchildren.

Upon the studies done, it was found out that grandmother was the second most frequent source of child care for women in employment [35]. In that, large number of pre-school children of working mothers uses grandmothers to look after these children during the absent-time of mothers. Almost the same amount of help and care are offered to school-aged children by grandmothers. However, this is a sort of exchange, i.e. while the daughter sees her visits as "keeping an eye on mom", mom may see the visits as the daughter turning to her for help and advice [36]. He stresses that to be on the receiving end in old age amounts to an abrupt reversal of the parental role.

Older people being the whole of a generation survived to a certain age, is the result of demographic changes in the population, i.e. the outcomes of longer life expectancies, lower birth rates, and an older average age of giving birth [37]. This scenario eventually leads to the phenomenon of grandparents. It has been recognized that such changes are likely to result in significant increases in elder abuse which includes grandparents too [38]. Sociologically speaking, disproportionate increases in the number of dependent elders (Grandparents) relative to working-age individuals may result in higher stress levels among caregivers and increase abuse opportunities and thus may act to increase the rates of grandparent's abuse.

Findings and the literature on elder mistreatment, and misbehaviour with the elderly appears to emphasize dependency and stress as two of the most significant factors. For example, a positive relationship between abuse and stressful workplace environment has been emphasized in Pillemer Finkelhor [39] and Pillemer \& Moor [40]. In many cases, the elder and grandparents' abuses are hidden, and as a normal and routine movementdepending on societies and cultures, poverty and affluence etc. For example, Harrington found a positive relationship between nurse staffing hours and nursing home deficiencies. The specific mechanisms that lead to increased levels of abuse, neglect, and exploitation, are not clear, what is consistent among the studies is that, a decrease in the qualified workforce is inversely related to abuse [41,42].

Similarly, safety and quality of life of grandparents highly depends on health status and personality traits, and ethno-cultural backgrounds of those around such elderly people. Therefore, such people may abuse the elderly, or have good behaviour with them $[43,44]$. In the present paper, we are unable to uncover all the dimensions regarding the grandparents' lives because of data limitations.

\section{Conclusion}

The paper comes to the conclusion that Iran does not have enough number of social workers and care-givers to put up with the increasing number of the ageing grandparents. Increasing life expectancy in Iran means that couples would remain married for longer time, which would need more care and sympathy with them in the years to come. Under the present demographic change, the potential ageing grandparents would need more investment with respect to their care and support. While the quantity of grandparents is increasing, their quality of life too must improve in the current changing time. The paper concludes that in case of Iran, wives not much welcome the retirement of husbands due to ever presence at home, diminishing of family's income and intervention of husbands in daily family routines etc. Similarly, loneliness followed by the death of a spouse is always a hard time. The research provided the opportunity to appraise variables such as age, the state of life, welfare, insurance, safety, education and ... of the elderly grandparents and within them. The present research includes a research design involving identification and the selection of research problem. In the empirical part of the research, the researcher could find more grandmothers than grandfathers; a sex ratio of 67.7 men against every 100 women.

According to UN predictions the elderly people will outnumber those under the age of 15 by the year 2050 in Iran-a situation that will need further social work services. Under the conditions of industrialization and urbanization, the aging grandparents need further protection and supports. In such a projected population change, increasing problems will appear within the elderly grandparents. As found out, families try to help and support the elderly and grandparents in Iran to improve their quality of life, but such help/services are not adequate. Therefore, such a great number of the upcoming generation of grandparents would need more invention rather than imitation. While grandparents benefit their grandchildren, they are benefited by them too in various ways. Hence, the youth must learn how to value grandparents not only in our society, but in any other society too. They can learn old culture and history from them.

Similarly, not all the elderly grandparents are in a state of safety, some face challenges associated with mental health problems, Alzheimer's disease, dementia etc. For instance, 44.12 percent of the sample grandfathers in the present study showed impatience. Depression among them is also on increase in modern time. The author came to know that in Iran, the quality of life of grandparents is widely influenced by the two spouses being alive; and if not the surviving spouse would feel increasing failures/shortcomings.

\section{References}

1. World population data sheet (2016) PRB. Washington DC, USA.

2. Cox H (1984) Later life: The realities of ageing. Englewood Cliffs, Prentice-Hall, New Jersey, USA.

3. Census of population and housing (2011) Statistical Centre of Iran.

4. Callahan D (1986) Adequate health care and ageing society: Are they morally compatible? Daedalus - The Ageing Society 115(1): 247-267.

5. Kosberg JI (1983) Importance of attitudes on interaction between health-care providers and geriatric populations. Interdisciplinary Topics in Gerontology 17: 132-143.

6. Marshall M (1990) Social work with old people. Macmillan. London, p. 25. 
7. Asefi A (1973) Today's grandfathers and grandmothers. In: Fern (Ed.), Anjoman Oliya va Morabian Publications, Izamber, Tehran, p. 16.

8. Jarrett RL (1994) Living poor: Family life among single parent African American women. Social Problems 41(1): 30-49.

9. Kalish R (1982) Late adulthood: Perspectives on human development. ( $2^{\text {nd }}$ edn), Monterey, California, Brooks, Seattle, Washington, USA.

10. Berado FM (1970) Survivorship and social isolation: The case of the widower. The Family Coordinator 19(1): 11-25.

11. (2007) Country Report, UN, ESCAP high-level meeting on the regional review of the Madrid international plan of action on ageing (MIPAA), Macao, China, pp. 9-11.

12. Laslett P (1976) Societal development and ageing. In: Binstock RH, Shanas E (Eds.), Handbook of ageing and societal sciences, Van Nostrand Reinhold, New York, USA

13. Cylke FK (1993) The Environment. Harper Collins, New York, UK.

14. Lenski G, Lenski J (1995) Human societies: An introduction to macrosociology. ( $7^{\text {th }}$ edn), McGraw-Hill, New York, USA.

15. Baltes PB, Shaie KW (1974) The Myth of Twilight Years. Psychology Today $7(10): 35-39$.

16. Blundell J (2001) Active sociology. Essex, Longman, Harlow, UK.

17. Charness NH (1995) Psychological models of ageing: How, who, and what? A comment. Canadian Journal on Ageing 14(1): 67-73.

18. Ben-Porath Y (1967) The production of human capital and the life cycle of earning. Journal of Political Economy 75(4): 352-365.

19. Mince J (1974) Scooling, experience, and earnings. Columbia University Press, New York, USA.

20. Becker G (1964) A theoretical and empirical analysis, with special reference to education. University of Chicago Press, Illinois, USA.

21. Ogawa N (2003) Population ageing and its impact on the socio-economic system in Japan. Ageing in Japan 2003, Japan Ageing Research Centre. Tokyo, Japan

22. Holmes ER, Holmes LD (1995) Other Cultures. Elder Years (2 ${ }^{\text {nd }}$ edn) Thousand Oaks, SAGE, California, USA.

23. Chi I (2001) Commentary: Asian perspectives on sociological issues for the millennium. Canadian Journal on Ageing 20(S 1): 118-124.

24. DeLehr EC (1988) Today's and tomorrow's ageing in Latin America. In ageing around the world: A report on the president's symposium on ageing in tomorrow's world: An international perspective. Gerontological Society of America, Washington DC, USA.

25. Chee YK (2000) Elder care in Korea: The future is now. Ageing International 26(1-2): 25-37.
26. Oh KM, Warnes AM (2001) Care services for frail older people in South Korea. Ageing and Society 21 (Part 6): 701-720.

27. Apt NA (2002) Ageing and the changing role of the family and community: An African perspective. International Social Security Review 55 (1):3974.

28. Kertzer ID (1995) Towards a historical demography of ageing. In: Kertzer DI, Laslett P (Eds.), ageing in the past: Demography, society and old age. University of California Press, Berkeley, pp. 363-383.

29. American Association of Retired Persons (AARP) (1998) Ageing Everywhere. Washington, DC, USA.

30. Anderson B (2001) Scandinavian evidence on growth and age structure. Regional Studies 35(5): 377-390.

31. Bloom DE, Williamson JG (1998) Demographic transitions and economic miracles in emerging Asia. World Bank Economic Review 12(3): 419 455.

32. Blanchet D (1992) Does an ageing labour force call for large adjustment in training or wage policies? In: Johnson P, Zimmermann KF (Eds.), labour market in an ageing Europe. CEPR, Cambridge University Press, Hyderabad, Telangana, India.

33. Bengstone V, Robertson J (1985) Grandparenthood. Beverly Hills. Sage Publications, California, USA.

34. Victor C (1994) Old age in modern society. Chapman and Hall, London.

35. Martin J, Roberts J (1984) Women and employment: A lifetime perspective. HMSO, London.

36. Harris C (1969) The Family. Allen and Unwin. Crows Nest, Australia.

37. Becker GS (2004) Economic approaches to understanding families. Understanding and optimizing human development: from cells to patients to population 1038(1): 201-205

38. Voelker R (2002) Elder abuse and neglect. A new research topic: Journal of American Medical Association 288(18): 2254-2256.

39. Pillemer KA, Finkelhor D (1989) Causes of elder abuse-caregiver stress vs. problem relatives. Am J Orthopsychiatry 59(2): 179-187.

40. Pillemer KA, Moore DW (1989) Abuse of patients in nursing homes. Findings from a survey of staff. Gerontologist 29(3): 314-320.

41. Benoit HM (2008) Lecture delivered at the centre for addiction and mental health. University of Toronto, Ontario, Canada.

42. ESCAP Population Data Sheet (2011), ESCAP Publications, Bangkok, Thailand.

43. (2006) Results of the decennial population and housing censuses in Iran, 1956-2006.

44. World population data sheet (2015) PRB. Washington DC, USA. 